\title{
Civilisations
}

Revue internationale d'anthropologie et de sciences

humaines

42-1 | 1993

Amérique latine-Europe

\section{Pouvoirs communaux, notables villageois et interventions de l'état dans les Andes péruviennes}

Jacques Malengreau

\section{OpenEdition \\ Journals}

Édition électronique

URL : http://journals.openedition.org/civilisations/2086

DOI : 10.4000/civilisations. 2086

ISSN : 2032-0442

\section{Éditeur}

Institut de sociologie de l'Université Libre de Bruxelles

\section{Édition imprimée}

Date de publication : 1 novembre 1993

Pagination : 53-90

ISBN : 0009-8140

ISSN : 0009-8140

Référence électronique

Jacques Malengreau, «Pouvoirs communaux, notables villageois et interventions de l'état dans les Andes péruviennes », Civilisations [En ligne], 42-1 | 1993, mis en ligne le 30 novembre 1996, consulté le 20 avril 2019. URL : http://journals.openedition.org/civilisations/2086 ; DOI : 10.4000/ civilisations.2086

Ce document a été généré automatiquement le 20 avril 2019.

(c) Tous droits réservés 


\title{
Pouvoirs communaux, notables villageois et interventions de l'état dans les Andes péruviennes
}

\author{
Jacques Malengreau
}

1 Les communautés villageoises qui occupent une grande partie de l'espace andin sont généralement perçues ou considérées comme les héritières des sociétés qui peuplaient l'entièreté de cet espace au moment de l'invasion espagnole qui les affecta il y a à peu près 450 ans. Déjà, à cette époque, les ancêtres de ces communautés constituaient la base d'une pyramide étatique complexe. Ces dernières n'ont pas cessé depuis lors de faire partie d'ensembles sociaux hiérarchisés, tout en expérimentant des restructurations diverses liées aux politiques des États coloniaux et républicains et en réagissant à ces politiques. Ces communautés, d' "incaïques », puis « indiennes » et «indigènes », sont devenues « paysannes » et « andines »; elles inspirent même aujourd'hui les associations de migrants urbanisés, tout en servant de modèles à certains mouvements autonomistes qui remettent en question les structures étatiques telles qu'elles ont été façonnées par des groupes ethniques extérieurs depuis l'époque coloniale.

2 Les communautés rurales des Andes, tout en s'inscrivant dans un environnement politique étatique, n'en conservent pas moins une certaine autonomie et une organisation politique spécifique. Elles entretiennent par ailleurs des liens ambivalents à plus d'un titre, tant avec les représentants régionaux de l'État, qu'avec des couches sociales élitistes provinciales et locales ou avec des personnages locaux influents. Ces élites provinciales et ces personnalités locales ont, de leur côté, construit leurs propres fiefs et réseaux de clientèle, assez autonomes à la fois à l'égard de l'État et envers la communauté villageoise par rapport aux membres desquelles elles bénéficient de certains privilèges. La nature sociale même des communautés prédispose leurs membres à adopter un profil ambivalent tout en leur permettant, à la fois de remplir certaines obligations dérivées des rapports de force externes et de conserver, voire de recréer, des rapports sociaux autonomes, différents, et d'une certaine façon, résistants. 
3 Nous verrons dans cet article en quoi deux communautés andines contemporaines maintiennent une aire de pouvoir spécifique et comment elles reconstruisent ou adaptent les mécanismes décisionnels imposés par les organes de l'État péruvien. Il sera question principalement des manifestations collectives ayant acquis un certain degré d'institutionnalisation, mais également de certaines d'entre elles qui, à défaut d'institutionnalisation, n'en constituent pas moins l'expression d'une recherche d'autonomie politique. Par ailleurs, cet article envisagera comment des personnalités locales, les notables villageois, récupèrent à leur bénéfice les contradictions inhérentes aux rapports internes aux communautés ainsi que celles qui opposent celles-ci, non seulement au monde du marché, sur lequel je ne m'étendrai guère ici $^{1}$, mais surtout aux relations de pouvoir dans le monde environnant. Tant ces mêmes personnalités que les communautés impriment leur marque sociale, qui sera précisée, au rôle que l'État entend leur faire jouer. Nous verrons également les diverses interventions effectuées par l'État dans les deux communautés étudiées, ainsi que la manière dont elles se sont articulées ou opposées aux communautés locales. Nous tâcherons alors de savoir sous quelle forme et dans quelle mesure il est possible de parler d'articulation des niveaux d'emboitement politique local et central de la société dite nationale.

4 Cette étude repose essentiellement sur des observations réalisées lors de séjours prolongés que j'ai effectués dans deux communautés villageoises des Andes péruviennes au cours de diverses périodes s'étalant de 1967 à 1989, en m'éclairant également de la littérature sur le sujet ; elles prennent également en compte sans les traiter directement d'observations faites lors de séjours plus courts dans de nombreuses autres communautés andines des régions qui englobent ces deux communautés. La première communauté observée, principalement au cours de près de dix-huit mois de séjour dans le village même entre 1967 et 1970 et au cours de deux brèves périodes ultérieures en 1974 et 1987 est le village de Cusipata dans la vallée du Vilcanota au sud de la région de Cuzco. Les observations y sont donc principalement antérieures à la Réforme Agraire et aux lois du régime du général Velasco (1968-1975) sur la communauté paysanne. La seconde communauté est le village de San Carlos situé sur les versants orientaux du moyen Utcubamba au nord de Chachapoyas, dans le nord du pays, village observé au cours des années 1974-1975 et au cours de plusieurs séjours entre 1982 et 1989. pour une durée totale de près de dix-huit mois dans le village même également. Les observations y sont postérieures aux réformes vélasquistes. Mais les différences apparaissant entre les deux communautés ne peuvent être réduites à ce seul paramètre. Non seulement l'observation des processus de décision dans la vie quotidienne et les entretiens avec les habitants, mais également l'analyse des procès-verbaux des réunions des organes de pouvoir au cours des dernières décennies servent de matière aux considérations présentes.

5 Derrière les institutions imposées ou définies par l'État central, ce sont la pratique, la signification, mais également l'adaptation locale de ces institutions, qui nous renseignent sur les structures locales particulières d'autorité, et cela indépendamment de la volonté du législateur central ou extérieur. Le fonctionnement local concret des institutions politiques définies au niveau de l'État reflète par ailleurs les similitudes et les différences des structures sociales locales et régionales, ainsi que l'histoire particulière, de chacune des régions représentées par des entités sociales particulières, en l'occurrence San Carlos et Cusipata.

6 Il est hors de propos ici d'établir une comparaison systématique qui ne saurait être effectuée sur la base d'un nombre aussi limité d'unités sociales observées à des époques 
différentes et sans en aborder toute l'histoire complexe et différenciée, tâche difficilement réalisable. La comparaison sert ici essentiellement à susciter des observations qui n'apparaissent qu'en contrepoint, non seulement en regard de l'expérience du monde social et culturel d'où provient l'observateur, mais dans les particularités notées entre deux entités sociales relativement proches en regard de ce même monde d'où provient l'observateur, mais malgré tout spécifiques l'une par rapport à l'autre. Car les différences et similitudes entre les monde européen et américain ne sont elles-mêmes qu'illusoires si elles ne prennent pas en compte la diversité de chacun de ses mondes, et en ce qui nous concerne ici, du monde andin péruvien contemporain.

\section{Le contexte historique du pouvoir communal}

7 L'État central du Pérou, dans la foulée des mesures prises à l'époque coloniale, un moment mises entre parenthèse par les politiques post-indépendantistes et républicaines reflétant l'individualisme libéral du XIX siècle, a institutionnalisé une certaine autonomie de la structure sociale et politique communautaire villageoise indigène dans le cadre d'un rapport collectif à une portion déterminée de territoire ; ce statut est garanti à la communauté paysanne par une disposition de la Constitution péruvienne de 1920 . Mais, une entité villageoise doit cependant être nommément désignée et reconnue légalement en tant que communauté par le gouvernement central avant de bénéficier du statut qui lui est garanti par la constitution. La loi sur les communautés paysannes élaborée cinquante ans plus tard par le régime du général Velasco vient préciser le droit constitutionnel et le droit coutumier en la matière, tout en imposant à la communauté des formes décisionnelles inspirées de modèles démocratiques extérieurs. Ces dispositions constitutionnelles et légales sont encore d'application, malgré les menaces récentes mais sans effet réel de démembrement institutionnel communautaire. C'est ainsi que la communauté villageoise, « indigène » jusqu'en 1970, «paysanne » ensuite, dispose d'organes politiques distincts de ceux de la municipalité, qui constitue l'échelon inférieur de l'administration de l'État central. La communauté villageoise n'en n'a pas moins jamais échappé au contrôle de l'État central, et cela depuis avant l'invasion espagnole.

8 Il convient de souligner le fait que les institutions du pouvoir local avaient déjà connu d'importants changements au cours des périodes coloniale et républicaine, et cela, jusqu'à l'époque contemporaine, avant les mesures prises par le régime vélasquiste. Ce dernier visait à renforcer l'organisation des communautés autochtones dans leurs limites territoriales du moment face aux propriétaires fonciers individuels. Le régime avait également comme objectif la modernisation et l'insertion de la paysannerie des communautés villageoises dans un projet d'intégration nationaliste et populaire, ainsi que la transformation en coopératives modernes des domaines fonciers et de leur maind'oeuvre journalière et permanente. À Cusipata, dans le sud, cette réforme n'affecta pas réellement la communauté, dans la mesure où celle-ci n'était ni ne fut reconnue à l'époque de la réforme, les autorités locales n'en ayant pas fait la demande. Le vent de la réforme ne s'en fit pas moins sentir sur les rapports entretenus entre eux par les habitants, en atténuant les barrières sociales qui les séparaient, et surtout sur les rapports entretenus entre ces derniers et les propriétés foncières voisines, en changeant profondément la nature des rapports sociaux au sein de celles-ci. La communauté de San Carlos, dans le nord, légalement reconnue de son côté depuis 1949, a appliqué ces réformes, tout en établissant son propre règlement interne. La Réforme Agraire 
proprement dite n'y eut pas de conséquence particulière, compte tenu de l'absence de grands domaines fonciers dans la région. Par contre. la réforme institutionnelle de la communauté qui suivit la Réforme Agraire y fut l'occasion d'un débat informel tant sur la nature que sur l'opportunité de l'organisation communautaire. Ce débat déborda donc le monde relativement fermé des militaires alors au pouvoir, des enseignants et des employés des dépendances administratives de l'État, qui en furent à l'origine. Comme à Cusipata, il s'y inscrit également dans la situation de crise provoquée par l'insertion accrue des membres des communautés paysannes dans les rapports centrés sur les villes et les centres d'emploi extérieurs.

\section{Le pouvoir local institué par l'État}

Comme ailleurs dans les Andes péruviennes, il y a à Cusipata ainsi qu'à San Carlos, plusieurs postes de pouvoir institutionnel, chacun lié en principe à des compétences spécifiques. Il y a ainsi le gobernador ou représentant local direct de l'autorité centrale, chargé de veiller au respect des lois nationales, des décisions des autorités locales et du maintien de l'ordre public, avec l'appui si nécessaire de la Guardia Civil ou gendarmerie, dont aucun des deux villages n'a un poste sur place. Il applique la loi nationale en fonction des conditions particulières locales. Le juez de paz ou le représentant de la justice sert d'arbitre dans les conflits qui opposent entre eux les villageois individuels et qui ne concernent pas les rapports à la terre et aux ressources naturelles, domaine réservé aux autorités en charge de la communauté ; il recourt également, en cas d'échec ou pour des actes ou des situations particulièrement graves, à ses supérieurs provinciaux du pouvoir judiciaire. Le conseil municipal, quant à lui, est chargé de l'administration locale des fonctions résidentielles, et en particulier du maintien du registre de la population, de l'entretien de la voirie du centre résidentiel, des bâtiments et lieux publics, de certains services urbains liés à l'église, à l'enseignement, à la santé, à l'hygiène et au confort urbain, notamment en matière de distribution d'eau ou de vagabondage d'animaux. Le cabildo abierto constitue l'assemblée des citoyens adultes, convoqués exceptionnellement par le conseil pour approuver les décisions et la gestion de ce dernier, en pratique plus que pour en débattre ou en décider. Depuis le début des années 1980, cet organe est devenu en théorie, davantage qu'en pratique, l'organe municipal de décision. Cusipata étant capitale d'un district comprenant plusieurs villages, son conseil municipal y exerce une autorité sur les autorités municipales des autres villages du district, et est à ce titre également conseil de district, selon le principe du primus inter pares en vigueur ailleurs dans les Andes; le conseil de district a la responsabilité des matières précisées plus haut relevant des différentes autorités municipales du district, mais s'occupe en fait surtout des services résidentiels relevant de la capitale de district. pour lesquels il fait appel à la coopération de la population de celle-ci, et secondairement de l'entièreté du district, les habitants des centres secondaires ne bénéficiant qu'occasionnellement des services du chef-lieu de district.

Selon mes informateurs locaux, le centre résidentiel de Cusipata était divisé, il y a quelques dizaines d'années en quatre quartiers, totalement effacés des rapports sociaux au moment de mes observations. San Carlos est quant à lui actuellement divisé en quatre quartiers appelés barrios, dont chacun a une certaine connotation parentale; chaque quartier a un responsable, appelé comisionado ; ce dernier est chargé d'assurer le relais du pouvoir exécutif entre les autorités municipales et la population du barrio dont il est 
responsable envers l'autorité municipale. À San Carlos, une dépendance résidentielle, Pedro Ruiz Gallo, datant du début des années 1960 s'est jointe à une dépendance résidentielle adjacente, Jazan, du district voisin de Cuispes pour constituer en 1981 un district séparé, détaché administrativement des deux villages centraux; comme il n'existe aucun autre village secondaire dans le district de San Carlos, le conseil municipal de celui-ci se confond avec le conseil de district et n'a en fait autorité que sur les habitants de sa propre aire résidentielle.

11 Il y a par ailleurs à San Carlos, mais non pas à Cusipata, où, rappelons-le, la communauté n'est pas reconnue légalement, l'assemblée des membres et le conseil de direction de la communauté, ce dernier émanant de l'assemblée. Les autorités communales sont chargées de veiller à la défense du territoire de la communauté, territoire auquel les habitants entretiennent non seulement un rapport de propriété collective, tant à l'égard des étrangers que de ses propres membres qui voudraient s'accaparer illicitement de certaines de ses portions, mais également un rapport d'identité fondé sur la mémoire d'une histoire et d'une ascendance collective. La communauté, par la voix de ces autorités, désigne, suspend, et peut même d'exclure ses membres; elle fixe son propre règlement interne ; elle fait par ailleurs effectuer des travaux d'aménagement de la voirie et des espaces fonciers de la communauté. C'est l'assemblée des membres qui prend les décisions. Le conseil de direction, nommé jusqu'à la fin des années 1980, conseil d'administration, se charge de préparer et d'exécuter les décisions de l'assemblée, tout en assurant leur suivi, et en veillant au respect des règlements communautaires ; c'est lui qui est chargé de résoudre les conflits qui peuvent se manifester autour des terres et des ressources naturelles de la communauté ${ }^{2}$ que ce soit entre des membres de celle-ci, ou entre la communauté et une personne morale ou physique extérieure à celle-ci. Le conseil de direction communal ainsi que l'assemblée dont il émane constituent, malgré le fait qu'ils s'intègrent dans un moule administratif externe, remis à jour au début des années 1970, l'autorité locale la plus autonome et la plus proche de l'ensemble des habitants du village et de leurs traditions spécifiques.

12 À Cusipata, la communauté n'étant pas légalement reconnue, le territoire n'est pas légalement reconnu comme étant propriété communale. Certaines de ses portions, cependant, essentiellement des pâturages, mais également des secteurs agricoles soumis à des normes collectives d'utilisation, en l'occurrence d'assolement, ainsi que les chemins, sont considérés par ses habitants comme extérieurs au domaine appropriable par des individus ; ces secteurs relèvent, à défaut de pouvoir communal, de l'autorité du conseil de district. C'est donc ce dernier qui en règle l'accès. Néanmoins, nous le verrons plus loin, des pratiques populaires locales conditionnent et limitent l'exercice du pouvoir de district dans certaines matières relevant de l'usage de l'eau et de la terre, tout en imposant un ordre social particulier autonome par rapport au pouvoir de district.

\section{L'interprétation et la construction locales du pouvoir}

13 Nous nous intéressons ici, au-delà des manifestations de pouvoir local officiellement reconnues par le pouvoir central, aux formes locales de fonctionnement de ce pouvoir local, ainsi qu'aux influences sociales et politiques particulières qui le sous-tendent ou qui le divisent, en établissant certains éléments de comparaison entre les deux villages qui ont davantage fait l'objet de mes recherches. Car, à Cusipata, comme à San Carlos, les 
conceptions et les pratiques locales de l'autorité conditionnent étroitement, mais pas forcément dans le même sens, le fonctionnement des pouvoirs officiellement établis.

Le gobernador, le juge de paix et le conseil municipal dans les villages-capitale de district, comme les agents municipaux qui les représentent chacun dans les hameaux secondaires, étaient, dans les Andes Péruviennes, jusqu'au début des années 1980, nommées par les autorités provinciales et confirmées par les autorités supérieures de l'État, et cela, chacun sur la base d'une liste limitée de noms présentées aux autorités provinciales par les autorités locales arrivant en bout de mandat. Ces fonctions constituaient donc non seulement les organes du pouvoir de l'État, mais reflétaient des forces politiques locales. Celles-ci construisaient leur pouvoir sur la cooptation, et donc à l'abri de tout contrôle direct de la population locale et sans aucune garantie publique de consultation populaire, à l'exception de la réunion occasionnelle d'une assemblée des citoyens, appelée cabildo abierto, rarement consultée et seulement dans des termes fixés par les autorités locales. Avec la restauration du pouvoir civil au niveau national au début des années 1980, pour la première fois, les autorités municipales, mais non le gobernador, ni le juge, seront élues tous les trois ans par la population sur la base de listes concurrentes élaborées, soit par les autorités locales, soit par certains opposants disposant de quelque influence dans le milieu local, voire régional. À San Carlos, où la communauté est reconnue depuis 1949, il y a un conseil communal qui est également élu tous les deux ans sur la base de telles listes depuis les réformes de la communauté paysanne du début des années 1970. Avant cela, comme ailleurs au Pérou, les autorités communales y étaient nommées selon la même procédure de cooptation qu'à cette époque les autres autorités locales.

Selon les procès-verbaux des réunions des autorités municipales et communales de San Carlos depuis plusieurs décennies, et selon mes observations et les dires de mes informateurs, des mêmes personnes en nombre restreint occupaient et occupent encore aujourd'hui successivement les différents postes d'autorité principaux, plus ou moins dans l'ordre, de chef de communauté à gobernador, de gobernador à alcalde ou juge, puis juge ou alcalde, pour occuper ensuite à nouveau l'une ou l'autre de ces positions. Ces mêmes personnes, lorsqu'elles ne remplissent pas une fonction officielle, fréquentent les réunions restreintes du pouvoir ; elles exercent là comme dans la vie sociale quotidienne du village une influence non négligeable; elles entretiennent également des contacts préférentiels avec les autorités provinciales. Une plus grande ouverture semble toujours avoir existé pour l'occupation des postes de chef, personero ou président de communauté, ou de gobernador, que pour ceux d'alcalde ou de juge, accessible à un nombre plus restreint de personnes, qui occupent ou réoccupent d'ailleurs parfois ces postes pendant de nombreuses années. Par ailleurs, un grand nombre de personnes, apparemment la majorité des hommes chefs de famille domestique, se succédaient et se succèdent toujours aux postes secondaires de conseillers municipaux ou communaux. Il existe donc une certaine hiérarchisation des postes correspondant à une certaine hiérarchie locale entre les villageois. Les personnages influents n'y constituent cependant pas une couche sociale fermée et exercent leur pouvoir en dehors de toute hérédité et de toute alliance, essentiellement en fonction de leurs capacités personnelles. Ils se sont parfois accaparé de certaines terres de manière relativement illicite, mais à titre de réserve ou comme moyen de spéculation, sans souvent disposer de la main-d'oeuvre nécessaire à leur mise en exploitation, ni dans la communauté, ni ailleurs. Ils ne sont pas nécessairement les gens les plus riches du village. Au contraire, les gros propriétaires de troupeaux préfèrent éviter les charges politiques, qui sont considérées comme occasionnant de lourdes 
dépenses personnelles et un frein aux ambitions matérielles, sans qu'elles ne permettent d'améliorer sensiblement une position économique privilégiée déjà acquise. Des gens moins aisés, au contraire, se font reconnaître socialement par l'occupation de postes politiques, qui dans certains cas, leur ont même permis de s'octroyer, parfois de manière détournée, des avantages matériels qu'ils n'avaient pas au départ.

Les informations orales et les procès-verbaux des conseils de district de Cusipata, où l'on ne retrouve pas d'autorité officielle de communauté, si ce n'est dans les hameaux annexes, y laissent apparaître une sélection plus tranchée qu'à San Carlos dans l'occupation des postes principaux d'autorité. À Cusipata, en effet, non seulement les postes principaux d'autorité, mais également les postes de conseillers municipaux ou de district, échappent en permanence à la plupart des chefs de famille, tandis qu'il n'existe pas de postes d'autorité communale reconnus. Seuls des postes subalternes d'assistants du gobernador et du juge y sont occupés par des personnes de la catégorie sociale inférieure des indigenas. La stratification sociale y apparaît de manière beaucoup plus nette, comme dans toute cette région du sud du Pérou. Les autorités y forment une couche sociale supérieure qui est composée de familles de notables, dont le statut supérieur s'acquiert de manière quasi héréditaire ${ }^{3}$ et dans lesquelles on n'entre pas si ce n'est par des mariages relativement peu fréquents d'une femme de statut inférieur avec un homme de statut supérieur; les notables sont désignés par le terme spécifique de vecinos, ou encore mistis ou caballeros, par opposition aux mestizos et aux indigenas qui y forment le gros de la population. Cette catégorie sociale, constituant près de $15 \%$ de la population, est également la plus aisée sur le plan matériel. Son maintien au pouvoir est facilité par le fait qu'elle forme un bloc relativement fermé, bien que parcouru de tensions et de compétitions personnelles, et que, contrairement à San Carlos, mais aussi aux autres entités sociales constituées en communautés, ses représentants au pouvoir n'ont pas de compte à rendre à une assemblée de communiers ou de citoyens.

Mais, semble-t-il, avant que Cusipata ne se constitue en district indépendant du district voisin de Quiquijana, en 1940, les rares notables de Cusipata exerçaient officiellement leur autorité à Quiquijana, l'ancienne capitale de district, en partageant les postes d'autorité avec les notables de cette dernière localité. Les notables de Cusipata étaient, selon les relations orales de ce village, des descendants directs de généraux créoles ayant échoué dans une tentative de rébellion ou de coup d'État national et ayant fuit pour venir se réfugier dans le village. La création en 1940 de postes nouveaux avec la constitution en district séparé a alors permis à certaines personnes des couches inférieures de joindre la strate des notables, par mariage ou par le pouvoir qu'il se sont forgé par des entreprises économiques individuelles, et d'occuper ces nouveaux postes avec les anciens notables. Ils en profitèrent pour confirmer voire améliorer leur statut social et matériel. Les notables ont ainsi inclus dans leurs rangs des habitants extérieurs à leur strate sociale, provenant de la couche sociale des indigena ou des immigrés venus d'autres régions, et qualifiés dans le village de mestizos à la fois par leur origine relativement modeste par leur niveau supérieur d'éducation ou par leur participation accrue aux activités commerciales. Mais, les vecinos ou notables de Cusipata prédominent au sein du pouvoir local. Comme à San Carlos, mais de manière plus appuyée et selon un mode plus héréditaire, des personnalités particulières exercent donc à Cusipata une influence continue dans les structures locales du pouvoir; et même lorsqu'ils n'occupent pas des postes officiels, ils servent de conseillers ou de "secrétaires" influents, ainsi que de relais difficilement évitable avec le pouvoir provincial. 

peu émoussée et ouverte depuis les années 1970 par l'accession de davantage de mestizos aux postes d'autorité, et cela suite à la Réforme Agraire qui a permis a un certain nombre d'habitants de prendre conscience de la possibilité d'alternatives en matière de relations sociales et de se lancer dans des activités à caractère commercial sur des haciendas voisines provisoirement délaissées par leurs propriétaires. Ces derniers tentaient il y a quelques années de reprendre le contrôle de leurs anciens domaines avec l'aide de certains fonctionnaires régionaux de l'État. les notables locaux se sont toujours opposés pour préserver leur espace de pouvoir, les notables de Cusipata règlent eux-mêmes les rapports individuels et collectifs à la terre, sur laquelle la pression démographique est beaucoup plus forte qu'à San Carlos; ils le font en veillant surtout à leurs prérogatives en la matière. Ils ont en effet souvent amélioré leur patrimoine foncier en s'emparant de terres d'autres villageois qu'ils avaient endettés en les obligeant de mettre leurs terres en gage. Ainsi, ce sont eux qui occupent les meilleures terres irriguées, tandis que leur position sociale privilégiée au sein du district leur permet d'établir des relations dominantes de type clientéliste avec les autres habitants du village, comme avec des habitants des communautés voisines, écologiquement différenciées, pour avoir accès à leur main-d'oeuvre et à certaines de leurs terres dont la production est de nature complémentaire à la leur à Cusipata même $e^{4}$. pouvoir à San Carlos et à Cusipata. Si dans les deux cas, l'accès aux quelques postes principaux fait l'objet d'une sélection privilégiée, pour les autres postes, à Cusipata, la sélection est considéré à la fois comme un privilège permanent réservé à une élite, qui n'a pas de compte à rendre, sinon à ses supérieurs, et comme un acte d'exclusion à l'égard de la majorité des habitants, alors qu'à San Carlos, l'occupation de ces postes constitue une obligation morale et rotative pour la plupart des adultes masculins, ainsi qu'un moyen de pleine intégration à la communauté locale. À San Carlos, rares sont d'ailleurs les hommes qui n'occupent jamais de charge au cours de leur vie. Les autorités, sélectionnées davantage sur la base de critères de capacité et de moralité personnelles y représentent davantage la collectivité locale et recherchent son appui. Ceci n'empêche pas certains détournements de fonction; mais à la différence de ce que l'on observe à Cusipata, ces derniers sont fréquemment sanctionnés par des dénonciations et de la répression des autorités de tutelle. La reconnaissance communale et une stratification sociale beaucoup plus diluée différencient assez nettement San Carlos de Cusipata en matière de sélection pour l'occupation des postes de pouvoir. Mais néanmoins, jusqu'au début des années 1970, la nomination aux postes de responsabilité se faisaient sans élection, tant à San Carlos qu'à Cusipata, le procédé couramment utilisé étant la cooptation par les autorités locales en place de remplaçants proposés à la nomination des autorités de l'État. L'élection s'impose aujourd'hui partout au niveau des conseils communal et municipal.

L'introduction de l'élection dans le choix des autorités locales constitue donc une innovation, tant à San Carlos qu'à Cusipata. Elle implique, fait nouveau, la confrontation sur la place publique de positions différentes, mais surtout de réseaux différents de relations, avec à la clé la prédominance d'un clan social au détriment de l'autre, ce qui semble contraire aux pratiques de réciprocité, bien qu'asymétrique, qui constituent un idéal courant appliqué dans les deux villages.

Civilisations, 42-1 | 1993 
22 Mais l'élection ne rompt pas pour autant totalement dans son déroulement avec les pratiques antérieures. En effet, la sélection du personnel postulant des postes d'autorité locale répond dans les villages étudiés à des normes spécifiques de fonctionnement par rapport à celles qui prévalent au niveau de l'État national. D’abord, les équipes qui se présentent aux élections font toujours l'objet d'une cooptation par les personnalités locales les plus en vue, et cela tant à San Carlos qu'à Cusipata, comme il ressort d'observations personnelles faites à la fin des années 1980 dans les deux villages. Par ailleurs, à la différence de ce qui se passe au niveau national, où de très nombreuses listes se présentent avec des mots d'ordre différents, dans les villages observés, la présentation des candidatures implique une division duale des équipes en présence et ne se fait pas sur des programmes. Si les listes en compétition se présentent chacune officiellement sous l'étiquette d'un des grands partis politiques nationaux, elles représentent surtout deux groupes locaux s'opposant chacun autour de l'une ou l'autre personnalité locale en fonction de luttes d'influence locales qui sont souvent assez éloignées des préoccupations affichées sur le plan national par les partis que représentent ces listes.

À Cusipata, la ligne de division dans la présentation des deux listes semblent se faire davantage en fonction d'un clivage entre, d'une part des vecinos et d'autre part des mestizas, chacune ayant sa clientèle propre d'indigenas et de mestizas. Mais il s'agit là d'une appréciation portant sur quelques cas d'élections sur lesquelles je dispose de quelque information, n'ayant pu enquêter systématiquement sur les différentes élections à l'occasion de trop brefs séjours effectués dans le village après l'introduction des élections. Une dichotomie plus générale entre notables et indigenas, d'où sont issus de nombreux mestizas qui n'ont pas immigré à Cusipata, traduit elle-même à Cusipata deux conceptions différentes mais articulées de l'ordre social, entre un système de propriété individuelle de terres divisibles et accumulables et un système de rapports collectifs à la Pachamama ou Terre-Mère, vénérée dans le monde andin traditionnel. Une observation semble cependant s'imposer, les notables, tout en gardant une influence importante, semblent avoir perdu le monopole du pouvoir local au bénéfice de mestizos, qui semblent jouer un rôle important dans les changements intervenus à Cusipata, en rompant la traditionnelle dichotomie entre notables et indigenas, comme ils commençaient déjà à le faire à la fin des années 1960.

À San Carlos, pour laquelle je dispose d'informations plus complètes postérieures à l'introduction d'une pratique élective publique et formalisée, la communauté entend par ailleurs réduire, ou plus exactement reconnaître, l'opposition, en incorporant l'un ou l'autre membre de l'équipe qui a perdu les élections dans le conseil composé des gens qui les ont gagnées. Les habitants valorisent en effet la coopération et non la confrontation entre les deux listes, l'union entre les villageois et non les divisions. Les divisions existantes, celle, quadripartite, des barrios, ou celle, duale des listes, ont un aspect plus rituel ou administratif que politique ou idéologique; elles reflètent davantage une valorisation de la complémentarité, voire de l'opposition, que de la suprématie ou de l'exclusion. La dualité politique observée à San Carlos s'inscrirait dans une tradition ancienne de clientélisme : avant que n'existent les élections, en effet, deux personnalités ayant chacune une clientèle stable s'opposaient en effet de manière permanente à l'occasion de l'élaboration des décisions à caractère collectif concernant la communauté villageoise. Cette dualité des chefs aurait-elle existé également à une autre époque à Cusipata, où l'opposition de classes et de systèmes sociaux semblent aujourd'hui avoir le pas sur toute autre forme de différenciation? Les informations dont je dispose 
actuellement ne me permettent pas de l'affirmer ni de l'infirmer. Les mythes de fondation de la communauté de San Carlos expriment cependant l'importance primordiale de la dualité, notamment l'un d'entre eux qui se réfère à la confrontation entre un chef autochtone et un conquérant espagnol : les deux personnages antagonistes, dont aucun ne parvient à prendre le dessus, s'entretuent; une source d'eau jaillit alors du lieu du double sacrifice ; mais cette eau, normalement source de vie, répand la mort, entraînant ainsi la destruction par éclatement en deux partie du village originel, mais aussi par là même le déplacement d'une moitié et la reconstruction de la communauté actuelle, San Carlos, l'autre moitié étant à l'origine de la communauté actuelle de San Pablo. Des mythes impliquant des oppositions duales similaires et des variantes transformationnelles du mythe sommairement résumé ci-dessus existent dans tous les villages de la région que j'ai visités.

L'appréciation portée par la population sur les autorités locales est elle-même fort ambiguë, tant à Cusipata qu'à San Carlos. Elle implique un mélange de respect et de dénigrement. Les acteurs politiques impliqués dans ces confrontations sont, lorsqu'ils accèdent aux postes d'autorité, à la fois des recours potentiels dignes de respect et les responsables des problèmes locaux ou les boucs émissaires des infortunes collectives particulières ; on fait appel à eux pour résoudre ses conflits et servir d'intermédiaire par rapport au monde extérieur; on les soupçonne également, pas toujours sans fondement, d'utiliser leurs positions pour se procurer des avantages personnels, ou au mieux, de se comporter comme des irresponsables. L'attitude critique est cependant plus manifeste à San Carlos. La prise en charge de responsabilités politiques y est également davantage considérée comme une obligation et se fait par le biais d'une participation rotative impliquant de nombreux habitants à cette prise en charge. La concentration du pouvoir et de ses avantages, licites ou illicites, y est donc plus limitée. L'introduction des élections jointe à la réunion d'assemblées des habitants a par ailleurs créé à San Carlos la possibilité de contrecarrer le pouvoir des autorités principales. Celles-ci, selon de nombreux informateurs locaux. par-delà la rotation des charges, et à l'image de ce que l'on observe à Cusipata, au moins au début des années 1970, et peut-être après, exerçaient auparavant leur pouvoir de manière plus autoritaire, et cela y compris à l'aide de châtiments corporels, tout en se livrant à de nombreux abus d'autorité. De nombreuses terres enlevées alors illicitement par d'anciennes autorités à la communauté ou à des particuliers ont été récupérées après l'instauration de nouvelles possibilités d'élection et de contrôle. La stratification sociale plus rigide, bien qu'actuellement en voie d'assouplissement, de Cusipata a peut-être freiné le rôle joué par les nouvelles pratiques d'élections et de contrôle dans l'exercice du pouvoir local. À San Carlos même, et dans une moindre mesure, à Cusipata, la plus grande légitimité du pouvoir s'accompagne d'une moins grande autorité de celui-ci, dans la mesure où les abus possibles de pouvoir sont à la fois plus limités et plus directement sanctionnés. L'occupation de postes d'autorité revêt un aspect d'autant moins prestigieux et attire d'autant moins facilement des personnalités entreprenantes. Il en résulte un certain vide institutionnel.

En ce qui concerne la nature des compétences des divers organismes de pouvoir et attachées aux postes qui les composent, celles-ci ne correspondent pas exactement à celles qu'a voulu leur accorder le législateur national, qui s'inspire lui-même d'un droit étranger et de l'image particulière qu'il se fait des communautés paysannes andines. Dans la pratique, le partage des fonctions entre les divers postes d'autorité est également beaucoup moins marqué que ce qui est prévu par le législateur. Tant les décisions 
concernant des initiatives nouvelles spécifiques ou les modifications précises à apporter dans l'environnement public ou extradomestique, que la conciliation entre divers intérêts locaux et extralocaux, font l'objet de concertation, non seulement de l'ensemble des autorités locales, mais également des notables qui n'occupent pas forcément un poste officiel à un moment donné; des consultations populaires informelles ou formelles préalables viennent éventuellement compléter cette concertation. Ces consultations sont cependant beaucoup plus restreintes et plus formelles à Cusipata qu'à San Carlos.

Dans les deux villages, l'objet sur lequel portent les décisions ou les initiatives locales des autorités concernent tant des réalisations de nature essentiellement sociale et symbolique que les rapports à la terre et à ses ressources. Il s'agit plus particulièrement, sur le plan social et identitaire, de veiller à l'aménagement du centre résidentiel et des chemins en vue de permettre le déplacement des personnes et des biens, mais aussi et surtout d'affirmer l'identité du groupe local, et cela par l'aménagement de la place publique centrale, d'un terrain de sport où la collectivité s'exprime par une compétition avec des groupes ou de villages voisins, de l'église où sont célébrés les saints du village, de lieux de réunion des autorités ou des habitants, ainsi que, poste important du budget, des frais de représentation auprès des autorités supérieures et de réception de celles-ci par les autorités locales. Les décisions locales ont également trait aux procès concernant les biens, essentiellement immobiliers urbains, considérés comme collectifs, et qui ont une valeur au moins autant identitaire qu'utilitaire. L'entretien des bâtiments scolaires incombe surtout, dans les deux villages, aux parents d'élèves et aux professeurs. Cette activité, du point de vue de ceux qui s'y livrent, répond à des objectifs de reproduction et de changement social, et étend les limites des préoccupations identitaires à un monde beaucoup plus large.

Mais le rapport au sol et à ses ressources constitue un autre objet essentiel de l'action des autorités locales. A Cusipata, le conseil de district qui est chargé de faire entretenir les chemins, non seulement du milieu résidentiel, mais également de l'espace rural, ainsi que d'assurer un usage des parcelles d'exploitation agricole ou pécuaire qui soit conforme au droit coutumier local. Ce droit implique notamment pour les habitants, et seulement pour eux, le droit de passage, le droit de faire paître leurs troupeaux sur toute terre en jachère, ainsi que le respect de règles d'assolement collectif imposées sur certaines terres. A ce titre, le conseil de district y remplit le rôle qui, à San Carlos, ainsi que là où est reconnue légalement la communauté, est accompli par le conseil communal, et cela conformément aux instructions de l'assemblée des communiers, Nous verrons par ailleurs qu'à Cusipata la tradition locale règle le respect de ces règles ainsi que le fonctionnement du système d'irrigation, mais cela, en dehors des institutions formellement reconnues par les autorités de l'État, et dans le cadre d'une véritable manifestation non officielle de communauté. Différents droits collectifs sur le terre et les obligations qui y sont liées sont ainsi à charge à Cusipata du conseil de district ou conseil municipal, mais en fait, surtout garantis par une organisation communale informelle d'irrigation, dont les attitudes ne sont pas nécessairement conformes aux desiderata des notables. À San Carlos, les différents droits et obligations portant sur la terre et sur les ressources de l'environnement sont réglés et assurés par les organes politiques de la communauté légalement reconnue, à l'exception par contre de l'irrigation, pratiquement inexistante à cet endroit au climat plus uniformément humide dans l'espace et dans le temps. Mais surtout, à San Carlos, depuis le début des années 1950, ce sont l'ensemble des membres de la communauté, c'est-à-dire tous les chefs de famille qui, à travers l'assemblée des 
membres de la communauté, chargent les autorités de celle-ci, qui ne seront élues qu'à partir de 1971, de faire effectuer certaines tâches communes, et qui imposent leurs règles concernant le rapport des habitants au territoire communal, le centre résidentiel restant à charge du conseil municipal local.

Tant à San Carlos qu'à Cusipata, les décisions s'élaborent lentement par des consultations qui s'effectuent principalement en marge des organes officiels qui sont censés les prendre selon les dispositions légales nationales. La tradition constitue par ailleurs elle-même un cadre déterminant du fonctionnement des actes collectifs.

30 A Cusipata, en particulier, une catégorie limitée de personnes, les vecinos ou notables, participe en fait à ces consultations préalables à toute décision. Les vecinos se rencontrent lors de festivités familiales diverses qui les réunissent à l'écart du reste de la population dans le cadre des relations familiales et d'alliance qu'ils entretiennent plus volontiers entre eux. Les réunions des conseils de district ou municipaux constituent généralement le cadre où se formalisent les décisions sans que ne s'y manifestent clairement des oppositions. Quant aux réunions ouvertes des habitants, elles ont un caractère davantage cérémoniel que décisionnel et servent de procédé d'entérinement. Seules quelques personnalités notables ou mestizas y prennent la parole. Les indigenas qui, au cours de leur vie, ont fait preuve de générosité dans la prise en charge d'un certain nombre de célébrations rituelles de "saints » jouissent dans la collectivité d'un prestige particulier. Cela ne leur donne cependant guère de pouvoir au niveau des institutions politiques locales officielles. Ils exercent cependant une ascendance particulière dans le domaine des activités religieuses reconnues et encadrées par l'Église, mais se voient également reconnaitre une autorité particulière dans tous les problèmes de la vie sociale courante, et je le soupçonne, bien que cela restait très clandestin et difficile à établir de manière sûre au moment de l'observation, dans des pratiques clandestines de rapport avec les ancêtres et les êtres spirituels du monde autochtone, dont les "saints" ne semblent souvent être que des représentations. Les gens les désignent d'ailleurs parfois sous le terme de vecinos indigenas. Compte tenu de la rotation de la prise en charge des célébrations, un grand nombre d'indigenas, bien qu'ils soient beaucoup plus nombreux que les vecinos, occupent cette position à la fin de leur vie, ce qui témoigne d'une plus grande ouverture dans l'accès aux postes dirigeants dans le monde communautaire autochtone que dans celui qu'imposent les notables principaux au niveau de l'ensemble des citoyens du district formant, officiellement du moins, l'échelon inférieur de l'État. Les notables, quant à eux, exercent leur pouvoir aux postes officiels sans pour autant remplir ces charges rituelles, réservées aux indigenas. Ils n'en célèbrent pas moins en famille et de leur côté des « saints » directement associés à chacune des familles, tout en encourageant par leur présence la célébration des « saints » associés à la communauté. Il faut souligner le fait que certaines matières font l'objet de débats qui échappent en partie au pouvoir officiel des notables du district; elles sont débattues, nous le verrons plus loin, dans des organes spécifiques plus proches d'un pouvoir collectif de type communautaire. L'ensemble des décisions sont prises en fait sur la présomption d'un consensus général.

31 Mais si les habitants, surtout indigenas, adoptent extérieurement une attitude résignée, soumise et déférente à l'égard des notables, en privé, par contre, et entre gens de confiance, surtout jeunes, ils n'hésitent pas à faire part de leurs griefs à leur égard, et de critiquer leurs abus d'autorité. Par ailleurs, les habitants font le plus souvent preuve de peu de zèle dans l'exécution des prestations demandées par les autorités. Les initiatives publiques prises par ces dernières sont d'ailleurs rares et, nous l'avons vu, surtout de 
portée symbolique, en dehors de l'aménagement des chemins ou de commandes adressées à des spécialistes payés par contrat pour la construction des lieux où s'exerce et se manifeste particulièrement l'autorité.

A San Carlos, le processus de décision suit à peu près le même chemin qu'à Cusipata, mais les débats préparatoires y débordent largement le cercle de quelques personnalités locales, même si ces dernières jouent un rôle prépondérant dans la préparation des décisions. Les décisions ne sont donc prises la plupart du temps que sur la base d'un consensus plus réel et plus unanime construit progressivement, souvent sous l'impulsion des responsables, et qui met du temps à s'élaborer ${ }^{5}$. L'autorité n'est donc conçue comme l'expression ni d'un savoir éclairé de notables, ni non plus comme celui d'une majorité contre une minorité, comme ce serait le cas dans un système démocratique tel qu'il est présenté par l'éthique politique européenne. Dans un tel contexte décisionnel, les innovations s'imposent difficilement ou le font sous la pression d'institutions ou d'événements extérieurs, ou en s'appuyant sur celles-ci. Et encore, dans ce dernier cas, en cas de contestation, les habitants manifestent un absentéisme plus élevé que d'habitude dans leur participation à l'exécution des décisions. Il arrive également, fait plus rare à Cusipata, qu'une personne se mette en congé de son poste d'autorité ou soit démise de celui-ci à l'initiative de certains habitants. Inversement, à San Carlos, mais non pas à Cusipata, les autorités, ne pouvant construire l'unanimité, en arrivent à mobiliser les membres de la communauté locale pour faire appliquer une décision publique par la force; c'est ainsi qu'en 1981 elles mobilisèrent tous les membres de la communauté sous la forme d'un service ponctuel obligatoire, dans le cadre de l'imposition en travail ou obligacion, pour faire démanteler des clôtures érigées illicitement par un groupe organisé d'habitants sur des terres communales, ou encore, dans les années 1980, elles constituèrent des patrouilles de villageois ou rondas campesinas, au sein desquelles les chefs de famille masculins furent réquisitionnés sous une forme rotative pour assurer notamment la protection du bétail contre les vols dont ce dernier faisait l'objet, Les autorités de Cusipata, trop strictement associées à une strate sociale locale restreinte, se trouvent, dans les cas d'oppositions qui dépassent le niveau individuel, obligées de faire appel à la force publique de l'État central, force que récuse, par contre le plus souvent, les autorités locales de San Carlos. Celles-ci semblent ainsi refléter une plus grande légitimité sociale que les autorités de Cusipata.

La légitimation consensuelle qui prédomine, et cela tant à Cusipata qu'à San Carlos, n'en n'exclut pas moins les oppositions, qui sont reconnues et même valorisées. Le consensus repose en effet, non pas sur la suprématie absolue d'une perspective, mais bien sur un équilibre partagé de positions différentes. Dans les conflits locaux, à Cusipata, comme à San Carlos, les autorités du village font fonction d'arbitres, davantage que de garants de lois ou de règlements, et recherchent essentiellement la conciliation, cela indépendamment d'un quelconque droit absolu, existant seulement dans le droit national, de chacun. Cette attitude semble exprimer la recherche d'un équilibre idéal entre éléments complémentaires, que l'on retrouve d'ailleurs dans la dualité des factions politiques; cet équilibre lui-même est étranger à une quelconque confrontation ou rupture permanente de l'ensemble concerné. A Cusipata, cette dualité se manifeste également de manière particulièrement inégalitaire dans les rapports entre personnes de catégories sociales différentes; mais si un notable s'impose à un indigena,le premier sert également d'arbitre dans un litige que le second aurait éventuellement avec un autre indigena, lui-même soutenu par un autre notable, auquel il est souvent lié par des liens de 
parenté rituelle. La prédominance d'un point de vue ou d'une cause se double donc, dans un cadre réciproque, d'une nécessaire reconnaissance de son opposé.

Ni à Cusipata, ni à San Carlos, les autorités ne sont considérées comme des spécialistes exemptés des autres fonctions sociales; elles ne sont pas rémunérées pour leurs fonctions ; mais dans les deux cas, et surtout à Cusipata, les gratifications personnelles à l'autorité à qui un habitant recourt sont les bienvenues, et même conseillées si ce dernier veut obtenir gain de cause. L'établissement de liens de parenté rituelle avec des personnages locaux influents est fréquent pour obtenir leurs bonnes grâces ; ces derniers s'y réfèrent pour faire appel à des services personnels divers et accroître leur prédominance sociale. Mais les personnalités locales doivent également honorer leur position de parrain et venir en aide à leurs filleuls ou protégés en cas de besoin. Cette attitude de générosité leur permet de justifier le pouvoir qu'ils exercent par ailleurs, ainsi que d'inscrire ce dernier dans un cadre de réciprocité. Ce type de rapports se manifeste davantage à Cusipata, où la différenciation sociale en termes de pouvoir est plus grande. Les détournements de certains biens publics à des fins privées ne sont pas rares et constituent une forme de rémunération qui est beaucoup moins bien acceptée par l'ensemble des habitants et qui résulte davantage de rapports de force. Ils sont plus limités à San Carlos où les autorités ne forment pas un groupe solidaire et permanent et où les abus entraînent plus facilement, mais non systématiquement, des dénonciations et des condamnations devant la justice extérieure au village.

\section{Notables villageois et citoyens locaux face à l'imposition communale et au contrôle des ressources}

Pour se constituer les instruments matériels nécessaires à l'exercice de leur pouvoir et à la réalisation de leurs projets, les autorités locales imposent les habitants; elles le font principalement et de manière ponctuelle en exigeant la fourniture par chaque chef de famille d'un temps fixe et limité en prestations gratuites en main-d'oeuvre; ce type d'imposition est désigné par le terme de faena ou cooperacion popular à Cusipata, et faena ou obligacion à San Carlos; l'imposition consiste également parfois pour chaque chef de famille à fournir une quantité fixe de matériaux locaux courants, et cela en vue de la réalisation d'un ouvrage spécifique et limité. Les autorités locales prélèvent également à des taux fixés d'avance des taxes diverses à la fourniture de certains services ou biens communaux, des amendes pour certaines infractions; elles prélèvent par ailleurs éventuellement de chacun des cotisations exceptionnelles. L'État central, de son côté, fournit, forfaitairement ou à des fins spécifiques selon les époques, certaines sommes limitées aux conseils municipaux pour l'exécution de travaux locaux d'intérêt collectif.

A San Carlos, les habitants participent chacun directement aux diverses formes de prestations aux autorités et se font assez rarement remplacer malgré un certain absentéisme aux prestations en travail et certaines négligences dans la remise des produits sollicités. A Cusipata, par contre, des mestizos, se font systématiquement remplacer à ces tâches obligatoires par des indigenas qu'ils rémunèrent pour ce faire, ou en payant l'amende, équivalent à une journée de travail, c'est-à-dire moins que le manque-à-gagner qui leur serait occasionné par leur participation à la faena; les vecinos, quant à eux, s'estiment exemptés de ces obligations en paiement de leurs responsabilités ; seuls les indigenas participent en fait couramment à l'imposition en travail. Il y a donc à 
Cusipata trois catégories sociales dont la solidarité par rapport aux biens collectifs ne franchit guère les frontières, alors que les habitants de San Carlos participent plus uniformément, si non dans des proportions supérieures, aux obligations collectives. L'absentéisme n'en est pas moins élevé dans les deux villages; il est cependant très difficile à apprécier, compte tenu du fait qu'il existe des prestations de substitution pour les absents, ainsi qu'un certain étalement dans le temps comme dans l'espace de l'exécution des prestations. Cet absentéisme ne traduit pas une simple négligence, mais semble refléter également un manque d'enthousiasme à l'égard de projets dont les aboutissements sont incertains et dépendent des autorités en qui la confiance est mesurée ; c'est particulièrement le cas pour des projets économiques, peu courants, mais dont les bénéfices ne sont pas assurés pour tous, quant ils ne sont pas indûment réservés au cercle étroit des autorités et des notables villageois.

A Cusipata, certaines réalisations collectives suscitent davantage que d'autres la participation. Ces réalisations échappent en fait au pouvoir officiel; elles sont le lieu d'une organisation plus spontanée et plus populaire des rapports sociaux; leur mise en oeuvre implique à la fois une hiérarchisation des habitants et une solidarité active entre eux dans leur réalisation. À cette occasion, les chefs de famille participants imposent leur propre ordre social, y compris aux notables du village. On voit alors fonctionner un ordre communautaire dépouillé des normes juridiques dans lesquelles l'enferme parfois sa reconnaissance légale nationale. Contrairement à ce qui se passe dans les communautés légalement reconnues, cet ordre ne concerne que l'usage de certaines ressources déterminées autre que la terre elle-même.

Cet ordre communautaire informel de Cusipata se manifeste en particulier dans l'aménagement du système d'irrigation et dans la répartition de l'eau d'irrigation ${ }^{6} U n e$ communauté officieuse des usagers de l'irrigation joue alors un rôle tout à fait essentiel dans la reproduction d'une partie importante de la terre cultivée. Un comité spécial permanent, indépendant des autorités de district, la junta de aguas ou " comité des eaux ", est chargé d'organiser le nettoyage des canaux d'irrigation à date fixe chaque année, le 28 juillet ${ }^{7}$, et cela avant le labour qui précède les semailles des produits qui bénéficient de l'irrigation. A cette occasion, l'ensemble des usagers de l'eau est réparti en deux groupes, chacun formé des personnes utilisant celui des deux systèmes de canalisation existant dans le village. Les gens qui font usage des deux systèmes doivent coopérer au nettoyage de chacun de ceux-ci, en travaillant successivement et doublement à l'entretien de chacun des deux systèmes. Chaque groupe élit son propre contremaître pour coordonner les travaux. Par ailleurs, au moment de l'irrigation, une assemblée réunissant les candidats du moment à l'usage de l'eau se rassemble quotidiennement à l'aube aux pieds des champs à irriguer. L'assemblée fixe alors les tours d'eau en fonction de l'ordre des demandes et éventuellement de certaines urgences qu'elle peut être amenée à reconnaître. Chaque candidat irrigue alors ses champs avec l'aide de la personne désignée pour lui succéder, celle-ci effectuant en même temps de la sorte un contrôle sur le bon usage de l'eau et sur le respect des tours fixés. A partir des années 1970, les vecinos, qui avaient l'habitude d'interrompre les tours d'eau décidés en assemblée sur simple notification préalable à celle-ci, furent contrés par les membres des assemblées qui les en empêchèrent. Les vecinos avaient d'ailleurs tenté en vain en 1960 d'organiser eux-mêmes la distribution en ménageant leurs propres intérêts. Mais le responsable nommé par les notables vendit les priorités aux candidats les plus offrant. Les habitants lui refusèrent 
alors tout crédit, et nièrent par là même toute autorité aux vecinos d'imposer leur ordre social, pourtant en vigueur dans les autres sphères du pouvoir.

Mais, à Cusipata, un certain contrôle dans l'usage de la terre, officiellement aux mains du conseil de district et des notables, s'effectue également sous une forme spontanée, voire clandestine, qui, ici encore, reflète une structure communautaire non officialisée. Ainsi, les habitants revendiquent leurs droits à la terre non tant comme objet de propriété, comme le font le droit national et les autorités notables qui le relaient, mais en fonction d'une relation personnelle de réciprocité avec la terre, conçue comme une personne liée à la collectivité locale. Dans le cadre de ce rapport de réciprocité, et indépendamment du droit national imposé par les autorités notables locales, le produit de la terre ne revient pas à son propriétaire, mais à celui qui est en interaction avec la terre. Ainsi, une plante qui n'aurait pas été récoltée et qui continue à croître reste liée à celui qui l'a plantée, même après que la parcelle sur laquelle elle se trouve ait changé de propriétaire ou d'usufructuaire. Les parcelles ne peuvent pas non plus faire l'objet de vente ou d'installations, plantations ou clôtures, qui en interdisent de manière permanente l'accès à d'autres habitants. Ces droits, de nature clandestine à Cusipata, où des actes de sabotage anonymes pénalisent ceux qui n'en tiennent pas compte, existent également à San Carlos, où ils se manifestent ouvertement et où ils sont sanctionnés publiquement par les représentants de l'assemblée communale et par la communauté.

Dans le cadre de ces rapports au milieu environnant, les indigenas et, dans une moindre mesure, les mestizos, à Cusipata, adressent leurs prières à la terre avant de la travailler, afin de la féconder. Ils célèbrent des fêtes à des saints, qui représentent sans doute également les esprits des ancêtres peuplant la montagne, ancêtres dont ils demandent l'intervention pour garantir la fécondité de la terre et des animaux. Ces fêtes sont collectives, familiales ou communautaires, et traduisent un rapport de solidarité à la terre et à ses ressources. Elles sont l'occasion d'un rapport redistributif entre les habitants, indigenas et mestizos, mais non vecinos qui, eux, assistent en spectateurs. Il convient de souligner en effet le fait que la prise en charge de célébrations de saints permet à une personne d'acquérir reconnaissance et prestige dans la communauté villageoise. Les chefs de famille sont moralement tenus de se succéder à tour de rôle à la célébration de chaque saint célébré dans le village, tandis que chacun des chefs de famille est censé célébrer les différents saints du village au cours de sa vie. Le nombre de célébrations prises en charge par une personne et le degré de générosité étalée à cette occasion par celle-ci fonde son prestige social dans la communauté. Ce dernier n'interfère cependant en aucune façon avec l'occupation des charges politiques réservées aux vecinos. Mais ses réalisations rituelles font d'un individu un interlocuteur privilégié tant des autorités politiques que du pouvoir ecclésiastique extérieur, et expliquent que les habitants le sollicitent pour faire usage de son ascendant sur le milieu social en général. A San Carlos, un système similaire de célébrations de saints fonctionne. Les saints y jouent également un rôle de fécondation de la terre et des troupeaux. Comme à Cusipata, ils procurent du prestige à ceux qui prennent en charge leur célébration, elle-même passage obligé de toute véritable reconnaissance sociale dans la communauté. Sans pour autant avoir de liens directs avec la sélection locale des autorités, cette prise en charge est un élément dont tiennent compte les électeurs pour apprécier les qualités des candidats qui se présentent à leurs suffrages. Elle joue donc indirectement un rôle politique plus important qu'à Cusipata, où les postes politiques locaux sont davantage monopolisés par une catégorie 
restreinte de gens, et où la générosité et la dévotion manifestée lors des fêtes de saints sous-tendent l'accès aux seules fonctions secondaires d'autorité réservées aux indigenas.

Selon mes informateurs locaux, certaines personnalités indigenas exerçaient les charges d'autorité principale du village avant la constitution de Cusipata en district séparé en 1940. Elles fondaient leurs décisions sur des assemblées d'habitants socialement moins différenciés. Les derniers indigenas à avoir occupé ces charges après 1940, et cela de manière symbolique, étaient des personnes qui avaient rempli de nombreuses charges de fêtes et étaient encore responsables au début des années 1970 de l'animation du culte catholique, de la réception du curé lors de ses visites et de l'entretien des biens de l'église du village. Ce sont eux qui se montraient les plus actifs défenseurs du rapport communal à la terre, et qui, dix ans auparavant, intervinrent avec succès auprès des autorités provinciales et étatiques pour qu'elles fassent restituer à la communauté des terres qui avaient été accaparées par une hacienda ou grande propriété voisine. Avant 1940, les quelques notables moins nombreux, qui exerçaient leurs fonctions politiques dans l'ancienne capitale de district, devaient avoir un statut plus proche de celui de moyens propriétaires, matériellement moins aisés qu'en 1970, et se maintenaient socialement à l'écart de la communauté locale, sur laquelle des autorités indigenas exerçaient davantage de pouvoir qu'en 1970. On ne peut cependant exclure le fait que les propriétaires fonciers et les notables de la région aient exercé une influence sur les décisions des autorités indigenas, ainsi que sur leur nomination par les autorités provinciales, comme ils le faisaient toujours au début des années 1970 en ce qui concerne les villages annexes et communautés légalement reconnues de Tintinco et Chillihuani.

Le pouvoir local, contrôlé par des notables villageois, fonde son autorité à Cusipata jusqu'au moins au début des années 1970, et peut-être par la suite, sur des liens privilégiés à la terre qui, depuis 1940, ne trouvent plus leur pendant dans les dépenses rituelles somptuaires auxquelles sont soumis particulièrement les indigenas. Il repose également sur son rôle de relais du pouvoir central. A San Carlos, le pouvoir local n'est pas lié à des familles particulières et, avec la reconnaissance légale de la communauté, il ne peut reposer sur des privilèges fonciers, si ce n'est de manière éphémère ; il constitue moins un relais de pouvoir d'État ou provincial qu'il ne concilie les forces politiques extérieures avec celles de la communauté villageoise; il recourt en fait rarement à des appuis du pouvoir central, si ce n'est, de la part de certaines autorités locales dans le cadre de détournements individuels de fonction.

\section{L'État et la communauté locale}

L'État se manifeste cependant couramment, tant à Cusipata qu'à San Carlos, et conditionne la réalité sociale de ces entités villageoises. Il le fait cependant rarement en interaction avec les autorités locales qu'il a toujours considérées davantage comme un instrument d'incorporation civique de la population que comme un partenaire d'entreprises ou d'actions collectives. A San Carlos, il est par contre davantage amené à dialoguer avec l'assemblée des communiers ou citoyens, dans la mesure où celle-ci exerce un contrôle réel sur les autorités locales et où il n'existe pas de couche sociale relativement organisée servant à la fois d'écran et de relais dominant entre le pouvoir et la base sociale, comme c'est le cas à Cusipata. permanent et qui marquent durablement les communautés villageoises étudiées de leur 
empreinte sont aujourd'hui, la reconnaissance et la délimitation des structures institutionnelles et de l'assise territoriale des populations locales, la conscription militaire nationale, l'enseignement primaire, certaines campagnes d'hygiène, en particulier de vaccination ou d'installation de réseaux d'eau de consommation, le financement de travaux d'urbanisation, souvent dans le cadre de rapports de clientèle, ainsi que le maintien d'un certain ordre social local lorsque sa rupture menace l'ordre national. Jusqu'en 1969, peu après l'installation du régime vélasquiste, un impôt foncier était également prélevé, ce dernier plus théorique que réel, dans la mesure où il est douteux que de nombreux propriétaires fonciers se soient effectivement acquittés de leur dû, et dans la mesure où la plupart des terres villageoises n'étaient pas inscrites au cadastre.

Si l'ascendance commune, l'adoption par la collectivité et le rapport commun à un territoire délimité détermine les frontières du groupe local ${ }^{8}$, l'État sanctionne ces frontières et situe le groupe dans la hiérarchie institutionnelle instaurée par l'État. Des personnalités originaires des villages pris en considération ici et qui exercent ou ont exercé des postes importants au niveau des structures étatiques ont joué et jouent encore un rôle actif dans le statut institutionnel des communautés villageoises dont il est question ici ; c'est plus particulièrement le cas à l'occasion de la perte de statut de capitale provinciale qu'expérimenta San Carlos en faveur de Jumbilla au début du siècle, comme dans la séparation en district autonome de Pedro Ruiz Gallo à l'égard de San Carlos en 1981, ou encore de Cusipata à l'égard de Quiquijana en 1940. L'État garantit également les frontières des entités locales et notamment des communautés : c'est ainsi que furent restituées aux habitants de Cusipata des parcelles accaparées par une hacienda voisine, ou encore qu'intervint la guardia civil ou gendarmerie pour séparer les communautés de San Carlos et de San Pablo qui s'affrontaient sur des terres limitrophes que chaque communauté revendique depuis plusieurs générations.

Le service militaire national obligatoire est l'occasion la plus courante pour les jeunes gens de faire l'expérience du monde extérieur. Comme les jeunes gens évitent fréquemment de se présenter spontanément à l'appel, à moins qu'ils ne désirent utiliser l'armée comme moyen de mobilité sociale dans le monde extérieur, la constitution des contingents fait souvent l'objet d'un recrutement forcé des jeunes gens, notamment par des rafles menées par la police à l'occasion des marchés ou des fêtes. Les jeunes hommes sont alors soumis à deux années d'entraînement militaire et d'embrigadement civique. Ils acquièrent parfois à l'armée certaines pratiques semi-professionnelles et un contact prolongé avec les pratiques et les valeurs du monde extérieur, tout en côtoyant, le plus souvent comme simples soldats, des personnes venues de divers coins du pays, généralement du milieu rural. Ils y forgent un sentiment d'appartenance nationale, mais y développent également de nouveaux désirs de consommation et de mobilité sociale. A San Carlos, en particulier, comme dans toute la région andine de Chachapoyas, et suivant en cela une tradition qui remonte à l'époque incaïque ${ }^{9}$, de nombreux hommes s'engagent volontairement dans une carrière militaire ou policière au service de l'État. Il s'est d'ailleurs installé à Pedro Ruiz, une caserne du génie qui a joué un rôle de pionnier dans l'ouverture de la route vers le bassin amazonien au cours des années 1970, et où de nombreux jeunes de San Carlos ont servi comme conscrits.

47 En matière d'enseignement, l'État central se manifeste au Pérou dans pratiquement toutes les communautés et tous les hameaux villageois depuis plusieurs dizaines d'années. A Cusipata, comme à San Carlos, il existe des écoles primaires, mixtes depuis les 
années 1970. A Pedro Ruiz, près de San Carlos, il existe même un collège secondaire desservant de nombreuses communautés de la région. Les nombreux jeunes de Cusipata qui suivent un enseignement secondaire se rendent dans la capitale provinciale ou départementale pour le faire et y séjournent toute la semaine. Tant à San Carlos qu'à Cusipata, l'école est le vecteur de normes patriotiques déterminées par l'élite aujourd'hui bourgeoise, largement créole et métisse, voire d'immigration relativement récente, de la capitale. La défense de la patrie et du territoire national et de ses symboles, notamment du drapeau national salué tous les matins par les écoliers sous la direction de leurs maitres, constitue un des thèmes scolaires favoris. Les murs des écoles arborent les portraits des héros nationaux créoles de l'Indépendance nationale. L'école valorise surtout la soumission à l'autorité, le travail urbain de bureau ou de commerce plus que l'entreprise créative ou les travaux manuels, ainsi que l'entreprise individuelle davantage que l'effort collectif. Les enseignants jettent souvent l'opprobre sur la vie locale traditionnelle, qu'ils jugent arriérée, et notamment sur les célébrations et les dépenses collectives communautaires liées aux fêtes de saints. L'école organise ses propres célébrations sans rapport avec les événements de la vie locale et aligne leur calendrier strictement sur les célébrations nationales. Même la mise en valeur du monde paysan andin s'effectue sur la base de représentations que se font de ce dernier, soit le pouvoir national en place, notamment à l'aide de symboles hérités d'une période incaïque mythifiée, souvent étrangers aux traditions locales, soit les mouvements critiques de l'ordre établi, qui se rangent derrière des directives révolutionnaires abstraites le plus souvent importées de l'étranger et le plus souvent déconnectées des valeurs paysannes indigènes.

Des associations de parents d'élèves ont été organisées par les enseignants pour tenter d'améliorer l'insertion de l'école dans le milieu social des enfants, mais souvent, et cela surtout à Cusipata, davantage pour faciliter la transmission des messages des maîtres aux parents que pour permettre à ces derniers d'influencer les enseignants. Les maîtres d'école jouent par ailleurs souvent, et cela dans les deux villages, un rôle permanent de conseiller auprès des autorités locales. Dans certains cas, au cours de la période sur laquelle portent mes observations, ils furent d'ailleurs originaires de familles locales, bien que davantage inspirés par les valeurs citadines de type élitiste. Malgré un absentéisme limité à certaines époques où les travaux agricoles et domestiques se font particulièrement pressants, très rares sont les enfants qui s'absentent régulièrement de l'école, celle-ci étant perçue par les parents comme un instrument potentiel de mobilité sociale par le biais des enfants. Les parents ont même ont souvent tendance à reprocher à tort ou à raison aux enseignants de ne pas en apprendre suffisamment à leurs enfants ou d'effectuer un enseignement dont ils ne comprennent pas l'utilité. Dans la région de San Carlos, de nombreux habitants ont d'ailleurs émigré dans la nouvelle bourgade de Pedro Ruiz qui s'est développé près de la route dans le fond de la vallée de la communauté de San Carlos pour y mettre leurs enfants au collège et pouvoir s'en occuper en même temps. Ils se consacrent alors à des pratiques commerciales et artisanales diverses pour subsister. Dans certains villages des régions abordées ici qui sont plus éloignés des voies de communication, l'absentéisme des élèves est par contre monnaie courante ; c'est là le fait également, dans une moindre mesure, des professeurs, dont les déplacements prolongés raccourcissent les séjours dans les villages, où ils ont souvent seuls la responsabilité de plusieurs classes ; ils y sont d'ailleurs envoyés non pas comme pionniers de l'éducation des populations rurales isolées, mais soit faute d'appui pour accéder à des postes plus confortables, soit à titre de sanction pour un état de service jugé déficient. 
49 A partir du début des années 1970 , les réformes éducatives du régime vélasquiste, sans pour autant remettre fondamentalement en question les quelques traits pédagogiques importants qui viennent d'être brossés, ont davantage mis en valeur l'éveil des jeunes aux réalités de leur environnement matériel et social immédiat et aux possibilités d'en développer les potentialités et ont modifié depuis quelque peu les perspectives éducatives. La scolarisation des femmes, jusque là négligée, a également été développée à partir de cette époque.

50 L'État contrôle et garantit en principe, le respect des lois qu'il édicte et de l'ordre social qu'il prétend instaurer au niveau national. Les dispositions qui concernent les rapports à la terre ou les atteintes graves à l'intégrité de l'État, sont susceptibles de temps à autre de justifier l'intervention de l'autorité supérieure, et plus fréquemment du pouvoir judiciaire. Cette intervention pouvant avoir lieu à la demande d'habitants particuliers, mais surtout à la demande d'autorités locales et dans le sens où elles le requièrent, le pouvoir et les privilèges de celles-ci s'en trouve renforcés. Des personnes plus influentes ou plus aisées n'hésitent pas parfois à dédommager des autorités pour qu'elles ignorent des affaires particulières illégales où les premières seraient impliquées. Cela arrive dans les cas de vols de bétail, phénomène courant qui porte son nom, l'abigeismo, dans les pâturages élevés de Cusipata comme de San Carlos. Bien qu'on peut observer lors de contacts directs entre des responsables villageois et des autorités locales que l'appui mutuel qu'ils s'accordent est parfois plus factice que réel, le fait que les autorités locales servent d'intermédiaires entre les habitants et les autorités provinciales ou supérieures donnent donc aux autorités locales un pouvoir accru sur leurs habitants. C'est d'ailleurs en grande pompe et avec une déférence particulière que les autorités politiques ou judiciaires supérieures sont reçues par les responsables villageois, à la fois à la vue des habitants locaux et sans la participation de ces derniers. A San Carlos, cependant, l'assemblée municipale ou l'assemblée communale, selon le cas, est généralement convoquée à cette occasion afin de donner son avis ou son approbation au motif de la visite, et cela indépendamment de la réception protocolaire des autorités. A Cusipata, un tel rassemblement aurait été exceptionnelle, en tous cas à l'époque où j'y fis l'essentiel de mes observations. Mais l'écart social entre les autorités locales et les autorités provinciales y était et semble y être resté également plus marqué qu'à San Carlos. Les secondes n'ont guère plus de considération pour les premières que souvent celles-ci pour les habitants du village, et surtout des villages dits «annexes ». Mais la menace, même factice, de l'intervention possible d'autorités extérieures à la requête des autorités locales permettent à celles-ci de se préserver certains privilèges. Cette menace est moins évidente à San Carlos, où les rapports entre certaines personnes locales et des personnalités extérieures peuvent garantir des services précis, pas nécessairement légaux ou licites, aux premiers, mais sans conférer un caractère permanent à leur autorité comme cela tend à être le cas à Cusipata.

51 A San Carlos, compte tenu de la reconnaissance légale de la communauté, les membres de celle-ci voient leur accès à la terre garanti et protégé de toute aliénation, au moins en principe, par les lois nationales et par les possibilités d'intervention d'un juge de terres régional qui est chargé de les faire appliquer. On y a cependant pu voir en 1981 des autorités régionales différentes prendre position pour des clans opposés dans un conflit foncier interne à la communauté, et cela sans qu'il soit tenu compte, dans le chef de certaines autorités de la région, des dispositions nationales légales en la matière. D'autre part des parcelles y ont été illégalement vendues, et cela avec à l'appui un contrat 
notarial public en bonne et due forme, et donc en infraction aux lois nationales et sans aucune intervention judiciaire.

Les personnes investies de pouvoir au niveau des institutions de l'État servent ainsi en milieu villageois des intérêts qui ne sont pas forcément ceux de l'État, ni ceux de la communauté légalement reconnue. Les divisions existant au sein même de l'appareil institutionnel de l'État sont susceptibles de mobiliser des forces locales antagonistes en rompant l'équilibre vers lequel tend la structure politique locale telle qu'elle a été définie plus haut. Mais à leur tour, les divisions locales conditionnent étroitement toute possibilité d'action de l'État à l'intérieur des communautés villageoises, où l'État est perçu en fait davantage comme un corps étranger avec qui il faut composer que comme une autorité supérieure à laquelle il convient de se soumettre.

L'État intervient également de manière ponctuelle dans des domaines relevant essentiellement de l'aménagement du milieu résidentiel urbain. Il s'agit surtout ici pour l'État, par ces interventions ponctuelles, de maintenir des liens symboliques pas trop coûteux financièrement avec les communautés locales. Le régime vélasquiste du début des années 1970, mais également, sous une forme plus électoraliste et moins dirigiste, le régime libéral qui lui a succédé dans les années 1980, ont développé un effort nouveau en la matière. Les budgets de l'État ont alors permis à Cusipata de reconstruire complètement sa place centrale en l'aménageant d'une fontaine et de quelques plantes, d'y reconstruire le local municipal sur la Plaza, d'installer un dispensaire, ainsi que de refaire le tracé de ses rues selon un modèle à angles droits prisé dans l'urbanisme colonial et moderne hispano-américain. A San Carlos, des nouveaux bâtiments ont été construits pour servir de locaux pour les conseils municipaux et communaux, et l'assemblée communale, de dispensaire et d'entrée au cimetière, tandis que l'église a été partiellement et l'école entièrement reconstruites, des portions de chemin recouverts de grosses dalles, et qu'a été complètement rénové un système de réservoir, de canalisation et de service d'eau, désormais fourni à domicile; un terrain de sport a également été aménagé, tandis qu'était rectifié le tronçon de route reliant San Carlos à Pedro Ruiz pour y faciliter le trafic motorisé. Tous ces travaux ont été effectué avec l'aide d'un financement de l'Etat utilisé conjointement avec certains apports locaux gratuits obligatoires en travail et en matériel. Mais le fait nouveau que constitue la subsidiation d'ouvrages publics par l'État dans les villages affaiblit la mobilisation sociale des habitants dans l'apport en main-d'oeuvre gratuite et facilite certaines formes de corruption au niveau des autorités locales.

Sur le plan médical, tant à Cusipata qu'à San Carlos, des campagnes occasionnelles de vaccination des personnes comme des gros animaux d'élevage ont été entreprises depuis quelques dizaines d'années. Ces campagnes ont été assez souvent maladroitement engagées et mal comprises. A San Carlos, en particulier, des négligences dues au personnel sanitaire dans la conservation des vaccins entraînèrent de nombreux échecs et accidents qui furent mis à charge des vaccins eux-mêmes par la population, quand ce ne fut pas à charge du gouvernement ou des étrangers fournisseurs du vaccin d'avoir voulu inoculer la maladie à la population. Mises à part les campagnes de vaccination, peu de couverture médicale est cependant assurée, si ce n'est, et cela seulement depuis le début des années 1980, la présence et les interventions limitées d'un infirmier ou d'une infirmière dans chacun des villages; ce personnel est payé par l'État pour ses services, mais vend les médicaments, qu'il doit se procurer lui-même. La plupart des interventions médicales antérieures étaient effectuées à l'initiative des habitants, de Cusipata, comme 
de San Carlos, tant auprès de guérisseurs locaux ou de passage, qu'à l'extérieur, auprès de médecins officiellement reconnus par les autorités nationales. L'amélioration hygiénique et l'extension de la distribution d'eau de consommation constitue à San Carlos une autre action effectuée à l'instigation du ministère de la santé. Mais elle s'étale sur plusieurs dizaines d'années, compte tenu du peu d'enthousiasme au départ de la part de la population pour coopérer à ce type d'installation, qui comprend un réservoir et de nombreuses canalisations, et dont les avantages n'ont pas été rapidement reconnus par les habitants. Ces derniers y voyaient surtout un moyen pour des employés du ministère de réaliser quelques affaires en vendant du matériel. Certains habitants encore craignaient voir inondées leurs maisons, comme dans des mythes d'origine du village. La communication avec les agents sanitaires de l'État mis du temps à s'établir.

Les institutions de l'État interviennent également ponctuellement dans des activités locales à caractère économique, tant à Cusipata qu'à San Carlos. Sauf au début des années 1970, époque du régime vélasquiste, l'intervention dans le domaine économique est resté limité à l'octroi individuel de crédit agropécuaire pour l'achat d'engrais et de semences améliorées, ainsi que dans l'amélioration des races animales d'élevage. Seuls quelques individus à la fois plus aisés sur le plan économique et plus entreprenants bénéficient généralement de ce type d'aide ; mais, faute d'assistance technique et de développement d'un système adéquat de commercialisation, les personnes aidées parviennent à peine à rembourser les prêts consentis et deviennent même souvent insolvables; elles sont alors obligées de revendre des animaux et de mettre leurs terres en gage pour apurer leurs dettes. Par contre, à la fin des années 1980, des prêts de la banque officielle à des habitants individuels de San Carlos portèrent leurs fruits, mais sous une forme imprévue, bien que prévisibles dans le contexte dans lequel furent octroyés les crédits. Ces derniers furent accordés avec l'appui du ministère de l'Agriculture pour améliorer des cultures de pomme de terre. Mais l'argent emprunté par des villageois à un taux d'intérêt inférieur au taux d'inflation, qui connaissait alors une augmentation galopante, fut utilisé par les emprunteurs à des activités commerciales de nature spéculative, et en particulier dans le commerce de bétail, leur permettant à la fois de rembourser les prêts et les intérêts et de faire du bénéfice. Une généreuse réception des employés du ministère désamorça les objections de ces derniers venus vérifier le bon usage des crédits en question.

Le régime vélasquiste est intervenu plus directement dans l'économie paysanne durant les années 1970, en recourant à des formes associatives nouvelles ou existantes. Elle le fit dans le cadre d'une réforme agraire qui affecta les grands domaines fonciers sans pour autant en redistribuer les terres aux communautés paysannes voisines qui les réclamaient, ainsi que dans le cadre d'une modernisation des structures légales de ces mêmes communautés paysannes. A Cusipata, l'intervention économique de l'État fut cependant pratiquement inexistante, tandis qu' à San Carlos, elle resta essentiellement limitée à un projet spécifique. Dans ce dernier village, le bureau régional du ministère de l'agriculture engagea en effet, non sans peine, la communauté, traditionnellement orientée surtout vers des activités d'autosubsistance domestique couplées à des opérations de troc ou de petite commercialisation occasionnelle et strictement domestique, à développer en entreprise communale une plantation d'arbres de type eucalyptus à des fins de commercialisation. Ces dernières entraient elles-mêmes dans les préoccupations qui étaient à l'époque celles du gouvernement de se créer une source nouvelle et plus accessible de matière première dans un grand nombre de communautés pour une fabrique de pâte à papier à installer dans une région voisine mais qui ne fut 
jamais construite. Après un premier échec lié à des études inadéquates du terrain, la plantation fut entreprise avec davantage de succès dans un autre secteur du territoire sous le contrôle d'un technicien du ministère de l'agriculture. Cette entreprise impliqua de nouvelles relations aux ressources de l'environnement et à la main-d'oeuvre. Des animaux furent d'abord déplacés d'un secteur traditionnellement destiné à l'élevage dans un secteur particulièrement propice à l'agriculture, puis de là dans des secteurs partiellement forestiers au détriment de leurs propres espèces végétales. Des journaliers du village furent payés pour leur main-d'oeuvre avec un prêt de l'État, consenti également pour acquitter la facture des pousses d'arbre; cela eut pour effet de déresponsabiliser partiellement la communauté locale, habituée à une imposition en travail dans les entreprises communales, à l'égard du projet. La commercialisation des arbres, arrivés à maturité après une quinzaine d'années sans s'être correctement développés, a permis le remboursement du prêt, mais cela grâce notamment à une forte inflation monétaire, et sans que la communauté ne réalise des bénéfices significatifs qui puissent être investis dans d'autres activités communales à caractère productif ou autres. L'éventualité de tels investissements ne fut d'ailleurs pas envisagée. L'État ne s'engagea pas non plus dans les villages concernés dans aucun projet coopératif qui n'eut pas comme cadre la communauté dans son ensemble. Il n'existe d'ailleurs dans les villages aucun groupe de production extradomestique en dehors des systèmes d'entraide liés à des rapports de réciprocité entre unités domestiques indépendantes.

57 A Cusipata, un réaménagement du système d'irrigation fut entrepris dans la cadre de la coopération bilatérale germano-péruvienne dans les années 1980, avec comme partenaire et bénéficiaire local, le village, représenté par ses autorités municipales qui, à défaut d'autorité communale légalement reconnue, assument également la charge de l'aménagement du territoire rural et non résidentiel. Le tracé initial du nouveau canal d'irrigation devait permettre d'étendre la surface irriguée totale du village. Il fut fait appel pour cela à l'imposition obligatoire en travail des citoyens du village. Mais le responsable péruvien local de l'administration de l'État modifia le projet en cours de route, cela pour improviser des économies sur le projet ainsi que pour contourner des passages qui se sont avérés plus difficiles à franchir que prévu, faute d'études préalables suffisantes. Le résultat est qu'aucune extension effective du système d'irrigation n'avait été obtenue lors de mon dernier bref séjour sur place en 1987, tandis que les corrections improvisées du canal étaient devenues la cause de dégâts systématiques causés au canal et aux champs voisins par la force du débit hydraulique.

58 L'État s'est toujours montré peu entreprenant en matière de réorganisation des circuits de distribution et de commercialisation dans le monde paysan, à l'exception de la parenthèse vélasquiste, durant laquelle des efforts particuliers furent entrepris en ce sens. Auparavant, les autorités régionales de l'État contraignaient les villageois à fournir des prestations gratuites pour l'entretien des chemins muletiers, qui étaient orientés vers les centres les plus importants, mais qui traversaient les divers villages de la région. Ces derniers ont été marginalisés par la construction à l'époque vélasquiste de la route dite «marginale » reliant la Côte Pacifique à la Forêt amazonienne et passant en bordure de l'ancien territoire de San Carlos. En effet, si elle rapprochait les villages des centres urbains, commerciaux et décisionnels, la nouvelle route a également, en facilitant le développement des échanges avec l'extérieur et en modifiant les règles de ces échanges, désorganisé les anciens circuits d'échanges régionaux relativement fermés. La politique de contrôle des prix des produits agricoles sur les marchés urbains par les différents 
gouvernements qui se sont suivis depuis le début des années 1970 vise à satisfaire la clientèle politique urbaine au détriment des revenus des petits producteurs, comme ceux de San Carlos ou de Cusipata. Elle a encouragé par ses subsides l'importation d'aliments, la dépendance nouvelle à l'égard de l'étranger en biens de consommation divers, tout en affaiblissant la production vivrière et en encourageant les activités spéculatives sur la terre et sur le bétail. L'hyperinflation qui s'est manifestée à la fin des années 1980 semble cependant avoir impliqué en même temps que la spéculation par certains, un certain repli sur une économie d'autosubsistance pour la plupart des villageois, les terres étant soumises à une exploitation à la fois plus extensive et plus intensive au niveau des unités domestiques locales.

59 L'État ne s'est guère intéressé non plus à la protection des ressources naturelles et s'oppose notamment dans la pratique aux directives communales de San Carlos qui visent à protéger les espèces rares de bois des forêts de la région. Ainsi, en 1982, lors de l'un de mes séjours sur place, le ministère de l'agriculture contraignait les autorités communales à laisser exploiter inconsidérément les bois locaux, pour permettre le prélèvement au bénéfice de l'État d'une taxe de $10 \%$ frappant l'exploitation du bois. De nombreuses espèces indispensables aux artisanats locaux disparaissent ainsi, accroissant la dépendance à l'égard de biens d'importation sur les prix desquels les habitants n'exercent aucun contrôle. Par ailleurs, le reboisement à base d'eucalyptus et de pins envisagé par les agents de l'État ne compense pas les pertes écologiques et économiques occasionnées par le déboisement sauvage. Les difficultés de communication permettent cependant de limiter les dégâts dus à l'attitude des commerçants et des agents gouvernementaux.

Les différentes interventions directes de l'État dans chacune des deux communautés sont de nature similaire. Comme activité permanente, elles portent essentiellement sur la conscription militaire et sur l'enseignement primaire, ces interventions étant surtout conçues sur le mode du développement d'une citoyenneté nationale et de l'encadrement politique de la population paysanne, et cela y compris à l'époque vélasquiste, qui s'intéressa cependant davantage à renforcer l'espace économique national sous le contrôle politique de l'armée, tout en utilisant les structures politiques autochtones existantes. Les autres interventions consistent surtout à subsidier l'aménagement résidentiel urbain, surtout dans ses aspects extérieurs et symboliques. Elles visent à intervenir ponctuellement et massivement sur la santé par des campagnes de vaccination et d'installation d'eau potable; elles facilitent ou garantissent l'octroi par les banques de crédits financiers aux individus les plus entreprenants dans des entreprises diverses de type agropécuaire, mais sans politique réelle d'aménagement du cadre social et économique de ces entreprises ou de leurs débouchés. Les interventions de l'État sur le plan économique sont particulièrement inefficaces, dans la mesure où elles sont souvent conçues dans des bureaux urbains, et ne tiennent compte en réalité, ni des systèmes de production, essentiellement domestiques, déjà existant dans les communautés, ni du caractère essentiellement non économique des entreprises collectives de la communauté, ni non plus, d'ailleurs, des nombreux blocages externes aux initiatives économiques nouvelles.

61 A Cusipata, ou l'ordre social est plus hiérarchisé, et dans une moindre mesure à San Carlos, les habitants font preuve d'une certaine déférence envers l'autorité, et surtout envers celle de l'État. Ils n'ont guère l'impression qu'ils peuvent influencer les décisions de l'État, si ce n'est sous la forme de requêtes, adressées par l'intermédiaire de liens de clientélisme politique avec certaines personnes originaires du village ayant acquis des 
positions privilégiées au sein des institutions de l'Etat. Souvent également, les villageois dénoncent la corruption, et au mieux l'irresponsabilité, de l'Etat, tout en déclarant pouvoir se passer de ce dernier. Cela dénote une attitude ambiguë assez conforme avec le caractère ambivalent des rapports politiques locaux.

Tant à San Carlos et à Cusipata, l'État, et c'est parfois également le cas en ce qui concerne le pouvoir notable local, est donc perçu à la fois comme un instrument de domination parasitaire et comme un père providentiel, mais surtout comme une entité abstraite, extérieure et étrangère au monde villageois local, et sur lequel il ne faut pas trop compter. Extérieurement cependant, les habitants de Cusipata, habitués de plus longue date et depuis l'époque préhispanique à des structures étatiques, manifestent une attitude rituellement plus déférente, mais pas pour autant dépendante, envers les représentants de l'État.

\section{Conclusions}

Dans l'ensemble, il existe un fossé entre les préoccupations de l'État et les pratiques et conceptions locales de l'ordre social et de l'autorité, et cela, davantage encore à Cusipata, où existe une catégorie sociale intermédiaire de notables, qui forme un écran au moins autant qu'un relais entre la population villageoise et l'État. Mais si l'encadrement politique des deux communautés villageoises abordées ici est largement conditionné dans sa structure officielle par ceux qui tiennent ou ont tenu les rênes de l'État au niveau national, il est surtout façonné par les populations locales en fonction de leurs structures sociales propres et de leurs traditions culturelles spécifiques. C'est ainsi que l'insertion plus ancienne de Cusipata dans une structure étatique, l'existence de propriétés foncières privées sur ses meilleures terres, ainsi que la stratification sociale plus rigide qui caractérise sa région impriment leur marque aux institutions politiques de ce village. Par ailleurs, dans les deux communautés villageoises, mais c'est davantage manifeste à San Carlos, où la reconnaissance de la communauté donne une part plus active aux habitants dans l'élaboration de leurs décisions collectives, le pouvoir s'exerce sur une base essentiellement consensuelle et à travers une lente élaboration; ce consensus n'exclut cependant pas les oppositions ; bien au contraire, il inclut l'opposition en établissant sur un mode dual une relation réciproque entre une position dominante et une position secondaire, mais sous une forme plus symbolique et idéologique que réelle à Cusipata. Le pouvoir est donc conçu et vécu comme la recherche d'un équilibre consensuel non homogène de positions inégales mais jamais ignorées. Le pouvoir de l'État national, davantage conçu sur un mode homogène et autoritaire, et où prédominent les rapports de force qui en sapent la légitimité auprès des populations locales, vient échouer sur ce mode autochtone de pouvoir, plus ou moins réel ou plus ou moins idéologique selon les situations abordées, et dont il tend à ignorer la légitimité propre autant qu'il en déséquilibre parfois les subtiles compositions.

Dans ce cadre, les relations entre les communautés locales et l'État n'ont pas seulement le caractère hiérarchique propre aux structures étatiques, mais se manifestent surtout sous la forme d'une frontière, tant dans le sens d'une disparité de sens ${ }^{10}$ que d'un espace local non entièrement absorbé par un État qui cherche à se construire, ni totalement contrôlé par la communauté, dont les manifestations tendent à être détournées par des autorités locales qui fondent leur pouvoir et le contrôle privilégié qu'ils exercent sur les ressources locales, naturelles et humaines, en partie sur une certaine double mystification de l'État 
et de la communauté. Les notables locaux, villageois, mais également provinciaux jouent en effet un rôle intermédiaire ambigu mais essentiel dans cette situation qui leur confère un pouvoir que seul l'État, ou seule la communauté, ne pourraient leur conférer.

\section{BIBLIOGRAPHIE}

BROUGERE, A.-M. 1986 « Transformaciones sociales y movilidad de las poblaciones en una comunidad deI Nor-Yauyos », in Bulletin de l'Institut Français d'Etudes Andines, 15, 1-2 : 133-158.

CARTER, W. \& ALBO, X. 1988 « La comunidad aymara : un mini-estado en conflicto » in ALBO, X. (compil.). Raices de América : El Nuevo Mundo aymara. Alianza Editorial. Madrid.

ESPINOZA SORIANO, W. 1987 Los Incas. Economia, Sociedad y Estado en la era del Tahuantinsuyo. Amaru Editores. Lima.

GUILLET, D. 1978 « The Supra-Household Sphere of Production in the Andean Peasant Economy » in Actes du XLIIe Congrès International des Américanistes (1976), vol. IV : 89-105.

MALENGREAU, J. 1972 Les Limites de la Communauté à Cusipata, un Village des Andes péruviennes. Thèse de doctorat en Sciences Sociales. Université Libre de Bruxelles. Bruxelles. 556 p.

1974 « Comuneros e impresarios en el intercambio : el caso de un pueblo cuzcefio ». in G. ALBERTI \& E. MAYER (Eds), Reciprocidad e intercambio en los Andes peruanos, Instituto de Estudios Peruanos, Lima, pp. 171-205.

1983a « Identifications et classifications socio-ethniques dans deux aires villageoises des Andes péruviennes », in Civilisations (Bruxelles), 23(2), pp. 187-226.

1983b «L'institution communautaire dans deux villages des Andes péruviennes », in Les Communautés rurales, Recueils de la Société Jean Bodin pour l'Histoire Comparative des Institutions, Dessain et Tolra, Paris, pp. 269-297.

1992a Espados institucionales en los Andes. Instituto de Estudios Peruanos.Coleccion minima 28. Lima, $104 \mathrm{p}$.

à paraître en 1993 : «Relaciones mercantiles de un mundo no mercantil en los Andes de Chachapoyas » (17 p.l. in Incorporation et désincorporation de la main-d'oeuvre indigène en Amérique latine. Sous la direction de J. RUIZ-GARCIA. Département d'Anthropologie et d'Ethnologie. Presses Universitaires de Vincennes. Paris.

MAYER. E. 1985 « Production zones » in MASUDA, S., I. SHIMADA, C. MORRIS eds, Andean Ecology and Civilization. University of Yokyo Press. Tokyo.

NELSON, G. 1986 « Migracion y estructuras sociales en una comunidad campesina : Cotahuasi » in Bulletin de l'Institut Français d'Etudes Andines 15, 1-2 : 159-175.

ORLOVE, B. 1991 « Mapping reeds and reading maps : the politics of representation in lake Titicaca »,in American Ethnologist, 18, 1 : 3-39.

PLAIT, T. 1988 « Pensamiento politico aymara » in J. ALBO, comp. Raices de América : El Nuevo Mundo aymara. Alianza Editorial.Madrid : 365-450. 
RASNAKE, R. 1988 Domination and Cultural Resistance : Authority and Power Among an Andean People. Duke University Press. Durham. N.C. 334 p.

SANCHEZ-PARGA, J. 1985 La trama del poder en la communidad andina. Centro Andino de Accion Popular. Quito. $456 \mathrm{p}$.

WACHTEL, N. 1990 Le retour des ancêtres. Les Indiens Urus de Bolivie XXe-XVe siècle. Essai d'histoire régressive. Gallimard, Bibl. des Sc. Hum. Paris. 689 p.

\section{NOTES}

1. Je renverrai à ce sujet le lecteur à d'autres publications que j'ai faites sur le sujet. Voir plus particulièrement en matière économique J. Malengreau (1974 et 1992b). Le lecteur consultera par ailleurs utilement pour plus de précision concernant des matières directement liées à l'exercice du pouvoir dans les deux communautés qui fournissent ici la matière première de mes réflexions, d'autres travaux personnels, où sont abordées plus en détail et de manière comparative, respectivement l'institution communautaire définie dans ses divers aspects (1983b), les catégories socioethniques telles qu'elles sont élaborées par la population dans les deux communautés (1983a), ainsi que le rapport à l'espace et à la terre dans les deux mêmes communautés (1992a: 9-40). Enfin, plusieurs idées reprises ici proviennent d'un ouvrage plus vaste que j'ai écrit sur Cusipata sous la forme d'une thèse de doctorat à l'Université Libre de Bruxelles(1972).

2. J'appelle indistinctement communal ou communautaire tout ce qui se réfère à l'organisation communautaire ou communale, distincte donc dans le cas présent de l'organisation municipale, qui dispose d'organes séparés.

3. Cette transmission héréditaire des postes principaux d'autorité par opposition à la succession rotative des postes subalternes d'autorité est mentionné pour les populations préhispaniques de ce qui constitue aujourd'hui les Andes boliviennes par T. Plati (1988: 376-379), et pour les populations andines boliviennes de l'époque coloniale, par R. Rasnake,(1988: 80-89), qui précise que l'extension des charges rotatives aux plus hauts postes diminua l'emprise de l'Etat colonial sur les populations indigènes, ce qui amena le pouvoir colonial à nommer directement de nouvelles autorités à ce niveau.

4. Voir pour plus de détails à ce sujet J. Malengreau, 1983a (202-205).

5. Le consensus et l'unanimité comme fondements décisionnels acquis par des consultations longues et répétées est attesté dans d'autres parties du monde andin autochtone de l'Équateur à la Bolivie. Voir à ce sujet, notamment, Carter, W. \& Albo, J. (1988 : 478), Guillet, D. (1976 : 95-96), Rasnake (1988 : 263) Sanchez-Parga, J. (1985: 154) et Wachtel, N. (1990: 125-126). Ces divers auteurs soulignent d'ailleurs la lenteur du processus décisionnel que cela implique.

6. On observe un phénomène similaire dans des communautés côtières où, malgré une privatisation poussée des terres, l'irrigation, rendue particulièrement stratégique par une grande aridité, constitue l'objet pratiquement exclusif de l'organisation communautaire. Voir à ce sujet E. Mayer (1985 : 55). Par ailleurs, là où on constate un important mouvement d'émigration vers les villes ainsi qu'un déclin des travaux en imposition publique, l'irrigation reste le domaine dans lequel l'imposition en travail se maintient le mieux. Voir à ce sujet A. -M. Brougere, 1986 : 139-145, et G. Nelson, $1986: 167-169$.

7. A noter que cette date correspond à celle de la Fête Nationale du Pérou, sans qu'un rapport quelconque puisse être établi avec celle-ci, seulement célébrée par les écoles du village.

8. Un ouvrage de l'auteur en préparation déterminera de manière plus précise comment les habitants définissent et articulent ces critères d'identité sociale à San Carlos, dans le cadre de l'emboîtement de groupes sociaux, territoriaux et identitaires à plusieurs niveaux. 
9. W. Espinoza Soriano (1987: 385-386) nous informe notamment de l'usage par les Incas de chachas dans la garde personnelle de l'Inca principal, cela pour démanteler les années chachas locales qui faisaient preuve d'une attitude rebelle à l'égard de l'empire.

10. Un travail récent de $\mathrm{B}$. Orlove (B. Orlove, 1991) est particulièrement éclairant à l'égard de la disparité des codes de communication entre des communautés paysannes de Puno, près du lac Titicaca et une agence de l'État péruvien, chargée à le fin des années 1970 de mettre sur pied un programme de réserve naturelle dans la région. L'auteur y confronte les cartes topographiques élaborées par des représentants des premières et celles qui ont été élaborées par des fonctionnaires étatiques dans la confrontation qui opposa l'État à certaines communautés paysannes locales. Le contenu et la forme de ces cartes varient beaucoup selon leurs auteurs, mais surtout, aucune des deux parties ne comprend ni n'essaye de comprendre la carte de l'autre tout en croyant à tort avoir imposé son point de vue, les cartes ne communiquant pas en fait en dehors du milieu restreint de chacune des parties dans lequel elles ont été élaborées et ne s'adressant pas en réalité dans leur conception à ceux à qui elles sont censées s'adresser.

\section{RÉSUMÉS}

Local Andean activities are analysed in this article with a comparative approach based on two Peruvian cases. one in the region of Cuzco. the other in that of Chachapoyas. The differences due to a differing history of insertion of hierarchical state structures can be observed particularly in the form of a more or less active participation of the inhabitants to the local authority' $s$ activities in each of the cases. Nevertheless, certain common characteristics appear: in particular, the search of a very wide compromise, based on the necessary ambivalent harmony between two factions which favours more one of them however. This situation is more often observed in the northem regions, less differenciated socially and less influenced by the State government. Relations between local communities and the State are comparable to frontiers, in the form of a disparity in mentality attributed to the relationship between govemors and govemed, as well as in the form of a shield composed of village notables, in their manipulating contradictions between the State govemment and the local authority.

\section{AUTEUR}

\section{JACQUES MALENGREAU}

FNRS, (ULB), Bruxelles 